\title{
IL LINGUAGGIO DELLA GIURISPRUDENZA DAL PUNTO DI VISTA DELLA FORMAZIONE DELLE PAROLE Orientamenti e problemi lessicologici
}

Con il presente lavoro si vuole contribuire alle ricerche sul linguaggio della giurisprudenza in chiave lessicologica. La ricerca si propone di mettere in rilievo i meccanismi della formazione delle parole nel linguaggio giuridico. La formazione delle parole è un procedimento vivo che rinnova il lessico e arricchisce la lingua con nuove unità lessicali. Il lessico si rigenera per vie interne mediante i meccanismi della composizione delle parole, della suffissazione e della prefissazione; $\grave{e}$ un corpus molto interessante dal punto di vista della formazione delle parole, è ricco di parole formate e di neologismi. Nel lessico della giurisprudenza convivono parole formate tradizionali e neologismi.

Keywords: formazione delle parole, linguaggio giuridico, lessicologia, neologia.

\section{Introduzione}

La formazione delle parole è intesa prima di tutto come studio lessicologico che mette in primo piano lo studio della neologia e dei neologismi, ottenuti con elementi esistenti nella lingua, che rinnovano per vie interne il patrimonio lessicale di una lingua. In tal modo la formazione delle parole è un procedimento vivo e produttivo.

La formazione delle parole è vista nella prospettiva sincronica; l'analisi lessicale si sviluppa in primo luogo dal punto di vista della consapevolezza linguistica dell'utente della lingua. Si analizzano solo i formati lessicali che nella consapevolezza linguistica dei parlanti di oggi sono sentiti motivati o trasparenti rispetto ad una base, per cui la base di un suffissato o di un prefissato è ciò che è sentito come vivo nella coscienza linguistica del parlante di oggi. Lo stesso criterio vale per le rispettive basi di un composto. Nel settore della formazione delle parole l'approccio sincronico e quello diacronico sono due obiettivi diversi che non vanno confusi. Il lessico di una lingua va inteso come fonte viva di cui possono disporre gli utenti di tale lingua; la distinzione non va fatta tra parole semplici e parole formate, munite di un altro elemento formativo, ma piuttosto tra le parole che costituiscono basi vive e che sono capaci di generare nuove unità lessicali, e parole sterili per la formazione lessicale perché non sono capaci di produrre nuove unità lessicali. La costruzione della parola formata deve essere motivata per il parlante di una data lingua. In tal modo sono soddisfatti i criteri lessicologici: i criteri semantici e quelli formali.

La formazione delle parole è un insieme di procedimenti formativi correlati tra di loro; tali procedimenti formativi, che corrispondono ad uno stesso meccanismo del linguaggio, sono oggetto della presente analisi che prende in esame il linguaggio della giurisprudenza. Le conclusioni sono provvisorie e cercano di suggerire qualche prospettiva di future ricerche in questo campo che saranno svolte sulla base di mate- 
riali più ampi e più completi. L'interesse dei linguisti per il settore della formazione delle parole, in particolare per le ricerche lessicologiche sincroniche che prendono in esame la fase moderna della lingua, è abbastanza scarso sia nel campo della linguistica descrittiva che nel campo della linguistica contrastiva. Non scarseggiano i lavori nella prospettiva storica e soprattutto $i$ lavori che analizzano le opere di alcuni autori dei secoli passati o delle correnti letterarie; sono studi stilistici in cui prevalgono le considerazioni stilistiche e letterarie. I settori della formazione delle parole poco frequentati dagli studi tradizionali sono i cosiddetti composti allogeni e i composti analitici. I composti allogeni sono formazioni lessicali ottenute con elementi greco-latini o con elementi stranieri; i composti greco-latini, e le altre formazioni lessicali che utilizzano gli elementi colti, entrano nella lingua e fungono da modelli per la creazione di altri neologismi. I composti analitici o le unità lessicali sintagmatiche sono formazioni lessicali costituite da due elementi formativi lessicali accostati ma non fusi o uniti per mezzo di una preposizione. Nella fase antica delle lingue romanze prevaleva la suffissazione, mentre nella fase moderna delle lingue romanze, e anche in italiano, prevale la composizione delle parole che arricchisce dall'interno la lingua italiana. Mediante la composizione delle parole si creano nuove unità lessicali formando serie di nuovi termini appartenenti a vari linguaggi tecnico-scientifici che poco a poco entrano nella lingua comune. Sociolinguisticamente parlando, queste unità lessicali colte, ottenute con elementi delle lingue latina e greca, parole di origine latina o greca, o formate modernamente sul modello delle parole latine, greche o di altre lingue, svolgono un ruolo di prestigio nella comunicazione linguistica, culturale e soprattutto nella comunicazione scientifica internazionale. Molte unità lessicali colte sono diventate internazionalismi. I composti colti svolgono un ruolo molto importante nello studio della neologia e dei neologismi.

La lingua italiana attinge dal lessico straniero per rinnovare dall'esterno il suo patrimonio lessicale. L'italiano si arricchisce con termini stranieri, in primo luogo inglesi: sono parole straniere, come killer 'assassino', o parole straniere adattate morfonologicamente, come verdetto, adattamento morfonologico dell'inglese verdict. Molte parole straniere sono diventate internazionali.

Il presente lavoro cerca di contribuire alle ricerche sul linguaggio della giurisprudenza dal punto di vista della formazione delle parole. Prende in esame la terminologia che riguarda il diritto pubblico e il diritto privato, e in particolare il diritto processuale penale, il diritto civile e il diritto del lavoro. La presente ricerca si propone di fornire la sistemazione dei materiali tratti da varie fonti - da dizionari dell'uso, stampa periodica, radiotrasmissioni e trasmissioni televisive; come risulta dall'esemplificazione riportata il lessico giuridico è molto interessante dal punto di vista della formazione delle parole, è ricco di parole formate e di neologismi. 


\section{I sostantivi formati}

La formazione dei sostantivi riguarda tre procedimenti formativi: la suffissazione, la prefissazione e la composizione delle parole nonché i rispettivi risultati di tali procedimenti formativi: i suffissati, i prefissati e i composti.

\subsection{I suffissati}

I suffissi servono a formare tre tipi di suffissati: i suffissati nominali o i sostantivi suffissati, i suffissati aggettivali o gli aggettivi suffissati e i suffissati verbali o i verbi suffissati. I suffissi si aggiungono alla base, cioè seguono la rispettiva base.

\subsubsection{I suffissati nominali}

Prima tratteremo i suffissati nominali ottenuti con suffissi mediante il procedimento di suffissazione; in tal modo si formano numerosi sostantivi suffissati da basi nominali, aggettivali e da quelle verbali. Se teniamo conto delle basi da cui i suffissati nominali derivano, cioè della categoria di partenza del procedimento di suffissazione, dei suffissi e della categoria di arrivo possiamo parlare di suffissati nominali denominali, di suffissati nominali deaggettivali e di suffissati nominali deverbali.

2.1.1.1. I sostantivi suffissati astratti ottenuti con il suffisso -tà e con le varianti morfonologiche -etàl-ità.

Numerosi sostantivi suffissati in -tà/-età/-ità derivano da basi aggettivali terminanti in -bile/-abile/-ibile: accertabilità 'possibilità di essere accertato' deriva dall'aggettivo accertabile, affidabilità 'condizione di minore che può essere dato in affidamento' deriva dall'aggettivo affidabile; il suffissato nominale alienabilità deriva da alienabile e annullabilità da annullabile. ${ }^{1}$ I sostantivi suffissati astratti in -tàl-etàl-ità derivano anche da basi aggettivali in -ale/-uale: conflittualità (da conflittuale), criminalità (da criminale), preterintenzionalità (da preterintenzionale), ${ }_{2}^{2}$ da basi aggettivali in -oso: criminosità (da criminoso), mafiosità (da mafioso), da aggettivi terminanti in -orio: obbligatorietà (da obbligatorio). Derivano anche da altri aggettivi che costituiscono basi vive dei sostantivi suffissati deaggettivali: giuridico in giuridicità, invalido in invalidità, recidivo in recidività. ${ }^{3}$

I suffissati nominali in -tàl-etàl-ità possono avere come base un prefissato: antigiuridico in antigiuridicità; dal prefissato antigiuridico si forma l'avverbio di modo o maniera in -mente: antigiuiridicamente 'in modo contrario al diritto'.

I suffissati nominali deaggettivali accertabilità, affidabilità, antigiuridicità, annullabilità, conflittualità, confrontabilità e mafiosità sono neologismi.

1 Cfr. altri nomi suffissati in -tà/-etàl-ità che derivano da aggettivi in -bile/-abile/-ibile: appellabilità, confrontabilità, imputabilità, inalienabilità, inammissibilità, inamovibilità, procedibilità, punibilità.

2 Dall'aggettivo preterintenzionale si forma l'avverbio di modo o maniera in -mente: preterintenzionalmente 'senza la volontà di uccidere'

3 Da giuridico, invalido e recidivo si formano gli avverbi di modo o maniera in -mente: giuridicamente 'secondo il diritto', 'dal punto di vista giuridico', invalidamente 'senza validità', recidivamente 'con recidività'. 
2.1.1.2. I suffissati nominali ottenuti con il suffisso -mento e con le varianti morfonologiche -amento e -imento.

Il suffissato nominale deverbale accompagnamento 'quello disposto dall'autorità giudiziaria per un imputato o un testimone' deriva dal verbo accompagnare; il suffissato nominale deverbale accreditamento 'procedimento con il quale un agente diplomatico viene investito del suo ufficio nello stato presso cui è inviato' deriva dal verbo parasintetico accreditare; il suffissato nominale deverbale imprigionamento ' 1 'imprigionare', 'il venir imprigionato' ha come base il verbo imprigionare. ${ }^{4}$

I suffissati nominali deverbali imprigionamento e accrescimento sono neologismi.

2.1.1.3. I sostantivi deverbali con suffisso zero

Il nome accusa 'imputazione' è un deverbale con suffisso zero (o senza suffisso) derivato dal verbo accusare; il nome arresto 'provvedimento limitativo della libertà personale' ha come base il verbo arrestare; il nome condanna 'provvedimento con il quale il giudice infligge una pena o impone un obligo' ha come base il verbo condannare; la base del nome deverbale spaccio 'vendita al pubblico di droga' è costituita dal verbo spacciare. ${ }^{5}$

2.1.1.4. I suffissati nominali ottenuti con il suffisso -tore e con le varianti morfonologiche -atore e -itore

I suffissati sono nomina agentis che derivano da basi verbali: il suffissato nominale accusatore 'chi denuncia e sostiene accusa' deriva dal verbo accusare; il suffissato nominale assicuratore 'ente che assume il rischio oggeto del contratto di assicurazione' deriva dal verbo assicurare; sequestratore 'chi compie un sequestro di persona' da sequestrare; spacciatore 'chi spaccia droga' da spacciare. ${ }^{6}$

\subsubsection{I suffissati nominali ottenuti con il suffisso-ista}

Sono prevalentemente nomi che indicano chi esercita la professione di ciò che dice la base; la base del suffissato nominale civilista 'giurista che si occupa di diritto civile' è costituita dal sintagma nominale diritto civile; la base del suffissato penalista 'esperto di diritto penale' e 'avvocato che si occupa di cause penali' è costituita dal sintagma nominale diritto penale o causa penale; la base del nome processualista 'studioso di diritto processuale' è costituita dal sintagma nominale diritto processuale. Il suffissato nominale cassazionista che ha come base il nome cassazione si riferisce all'avvocato iscritto nell'albo dei patrocinanti davanti alla Corte di Cassazione. Il suffissato nominale colpevolista che ha come base l'aggettivo colpevole si riferisce a chi, riguardo un processo si schiera con i sostenitori della colpevolezza dell'imputato. Il suffissato

4 Cfr. altri sostantivi suffissati in -mento/-amento/-imento che si riscontrano nella terminologia giuridica e derivano da basi verbali: accrescimento, adescamento, affidamento, annullamento, dibattimento, invalidamento, licenziamento, patteggiamento, perimento, procedimento, risarcimento, scarceramento.

5 Cfr. altri sostantivi deverbali senza suffisso: condono, confisca, confronto, delega, delibera, denuncia, divieto, indagine, indennizzo, prova.

6 Cfr. altri esempi: assolutore 'chi assolve', confiscatore 'chi confisca', confutatore 'chi confuta', donatore 'chi fa una donazione', patrocinatore 'chi patrocina', patteggiatore 'chi patteggia', punitore 'chi punisce'. 
nominale divorzista la cui base è costituita dal nome divorzio ha come significato 'chi sostiene l'introduzione del divorzio in ordinamenti giuridici' o 'esperto in cause di divorzio'. Il suffissato nominale processualista è un neologismo.

2.1.1.6. I suffissati nominali ottenuti con il suffisso -zione e con le varianti morfonologiche -azione e -izione

La base dei rispettivi suffissati è costituita di regola da un verbo: cassare in cassazione 'annullamento di un provvedimento giurisdizionale', colpevolizzare in colpevolizzazione 'il colpevolizzare, il colpevolizzarsi, il venire colpevolizzato', contravvenire in contravvenzione 'violazione della legge penale', delegificare in delegificazione 'provvedimento con cui si sottrae una determinata materia alla disciplina della legge e si trasferisce alla Pubblica Amministrazione il compito di regolarla', invalidare in invalidazione 'dichiarazione o dimostrazione d'invalidità', il verbo parasintetico scarcerare in scarcerazione 'liberazione dell' imputato sottoposto a custodia cautelare', o correntemente 'liberazione di un detenuto dal carcere'.?

I suffissati nominali deverbali colpevolizzazione, criminalizzazione e delegificazione sono neologismi.

\subsubsection{I suffissati nominali ottenuti con vari suffissi}

I sostantivi in -ario derivano da basi verbali: affidatario (da affidato) 'chi ha in affidamento qc. o qcn.', delegatario (da delegato) in diritto civile 'chi diviene creditore in seguito a una delegazione', deliberatario (da deliberato) 'chi in una vendita all'asta si è aggiudicato qualcosa', donatario (da donato) 'beneficiario di una donazione', sequestratario (da sequestrato) 'custode di beni sequestrati'. Da basi aggettivali si ottengono i suffissati nominali con il suffisso -ia non accentuato: delinquenza (da delinquente) 'criminalità'; incompetenza (da incompetente) 'mancanza di competenza'; soccombenza (da soccombente) 'condizione della parte soccombente'; perizia (da perito) nella procedura civile o penale 'consulenza, giudizio tecnico di un perito su una data questione'. I nomi colpevolezza e incolpevolezza, ottenuti con il suffisso -ezza, derivano da aggettivi: colpevolezza (da colpevole) 'condizione di chi è in colpa, di chi è colpevole', incolpevolezza (da incolpevole) 'mancanza di colpa'. I sostantivi in -ismo derivano da basi aggettivali e si connettono con i suffissati nominali in -ista: colpevolismo (da colpevole) 'atteggiamento di chi è colpevolista' e divorzismo (da divorzio) 'atteggiamento di chi è divorzista'. Il suffissato nominale deverbale affidatario, il suffissato nominale deaggettivale colpevolismo, il suffissato nominale denominale divorzismo e il suffissato nominale deaggettivale incolpevolezza sono neologismi.

Ecco ancora alcuni suffissati nominali: il suffisso -ale forma il sostantivo criminale 'delinquente' dalla base nominale crimine; il suffisso -iere forma il suffissato carce-

7 Cfr. altri esempi di suffissati in -zione che derivano da basi verbali: approvazione, assicurazione, assoluzione, carcerazione, confutazione, costituzione, delegazione, delibazione, deliberazione, detenzione, donazione, emanazione, impugnazione, imputazione, incriminazione, investigazione, istruzione, procedimentalizzazione, separazione. 
riere 'custode del carcere' dalla base nominale carcere; dal sostantivo prigione si hanno prigioniero 'chi è privato della libertà personale', formato con il suffisso-iero, e prigionia 'condizione di chi è prigioniero', formato con il suffisso -ia. Dal sostantivo avvocato si ha il suffissato avvocatura 'professione dell'avvocato', formato con il suffisso -ura. Il suffissato nominale interrogatorio 'complesso delle domande rivolte alle parti di un processo civile, all'imputato o al teste di un processo penale al fine di accertare la verità' si connette con il sostantivo interrogatore e con il verbo interrogare.

2.1.1.8. Si hanno alcuni aggettivi sostantivati: dall'aggettivo recidivo si ha il nome recidiva 'circostanza aggravante del reato che si applica a chi, dopo essere stato condannato definitivamente per un reato, ne commette un altro'; dall'aggettivo impugnativo si ha impugnativa; ${ }^{8}$ e dall'aggettivo scomparso ${ }^{9}$ si ha il nome scomparsa 'mancata comparsa di una persona, di cui non si hanno più notizie, nel luogo del suo domicilio o della sua residenza'. ${ }^{10}$

\subsection{I prefissati}

La prefissazione, procedimento formativo senza mutamento di categoria, serve a formare prefissati nominali, prefissati aggettivali e quelli verbali.

\subsubsection{I prefissati nominali}

I prefissi premessi a un sostantivo formano i sostantivi prefissati. I prefissi sono denominati anche affissi alla stessa stregua dei suffissi; si chiamano affissi gli elementi formativi che precedono o seguono la base. I prefissi che servono a formare i prefissati nominali sono contro-, in- con le varianti morfonologiche, ri-, semi-, sopra- e super-.

2.2.1.1. Il prefisso contro-, che indica opposizione, reazione, replica, forma i seguenti prefissati: controdenuncia 'denuncia presentata dal denunciato contro il denunciante'; controesame, 'interrogatorio di un imputato o di un testimone svolto dalla parte che in origine non ne ha chiesto l'ammissione'; controinterrogatorio 'interrogatorio della difesa o dell'accusa a un imputato o a un teste, in opposizione a quello già svolto dalla parte avversa; controparte 'parte avversaria in un giudizio civile o in una controversia'; controquerela 'querela che il querelato sporge a sua volta contro il querelante'; controricorso 'atto contrario al ricorso presentato dalla parte avversaria'. I prefissati nominali controesame e controricorso sono neologismi.

2.2.1.2. Il prefisso in-, che ha valore negativo, premesso al nome adempimento forma il prefissato nominale inadempimento 'mancata esecuzione della prestazione da

8 Il suffissato nominale deaggettivale impugnativa 'istanza con cui si propone un mezzo di impugnazione' è un neologismo.

9 Più precisamente dal participio passato del verbo scomparire, scomparso, usato come aggettivo; è un deverbale formalmente identico al participio passato del verbo scomparire.

10 Si trovano anche $\mathrm{i}$ participi presenti sostantivati come delinquente (derivazione da delinquere) 'chi ha commesso uno o più delitti', querelante (la base può essere sia querelare sia querela) 'chi sporge o ha sporto querela'. Più numerosi sono i participi passati sostantivati; alcuni esempi: accusato, affrancato, assicurato, carcerato, condannato, detenuto, imputato, indagato, indiziato, sequestrato. 
parte del debitore'; il prefisso $i l$-, che ha pure valore negativo ed è variante morfonologica del prefisso in-, premesso al nome liceità forma il prefissato nominale illiceità 'caratteristica o condizione di ciò che è illecito'.

2.2.1.3. Il prefisso $r i$-indica ripetizione nell'esempio di riesame 'riconsiderazione' che è un prefissato nominale; riesame è un neologismo.

2.2.1.4. Il prefisso semi- indica 'in parte', 'parzialmente' in semilibertà 'opportunità per detenuti di uscire dal carcere nelle ore diurne per lavorare e inserirsi nel contesto sociale'.

2.2.1.5. Il prefisso sopra- indica qualcosa che venga aggiunta a un'altra in sopraddazio o sovraddazio 'diritto suplementare pagato oltre al dazio ordinario'; indica superiorità di un'attività professionale in sopralluogo 'ispezione di luoghi disposta ed eseguita di persona dall'autorità giudiziaria per fini probatori'.

2.2.1.6. Il prefisso super- indica preminenza, superamento di un limite, eccesso e in particolare indica qualità superiore o condizione straordinaria, ma in supertestimone 'testimone che in istruttoria o nel corso di un processo è in grado di presentare prove decisive' indica qualità superiori o straordinarie del testimone; indica soprattutto l'importanza del testimone per il processo in corso, perché il testimone può aggiungere nuove prove che sono decisive per il processo. Il sostantivo prefissato supertestimone è un neologismo che si usa nel linguaggio giornalistico.

\subsection{I composti}

Se si confronta la fase moderna della lingua italiana con la fase antica si può vedere che la composizione delle parole non era propria dell'italiano antico, mentre la composizione delle parole è molto caratteristica dell'italiano di oggi; più precisamente sono nuovi tipi di composti: composti allogeni e composti analitici presenti nel lessico giuridico.

\subsubsection{I composti allogeni}

Sono parole formate ottenute con prefissoidi e suffissoidi, elementi formativi di origine latina e greca, che appartengono prevalentemente al linguaggio della scienza e della tecnica, ma molti sono entrati nella lingua comune.

2.3.1.1. I sostantivi composti ottenuti con un elemento colto

2.3.1.1.1. Il prefissoide auto-. Il sostantivo composto autodifesa, ottenuto con il prefissoide auto- 'di sé stesso', 'da solo' e con il nome difesa, indica attività difensiva esplicata personalmente come nell'esempio di autodifesa dell'imputato.

2.3.1.1.2. Il prefissoide micro-. Il sostantivo composto microcriminalità 'attività criminale di ambito locale, caratterizzata da reati di limitata gravità' è costituito dal prefissoide micro- che ha come significato 'che ha relazione con cose piccole' e dalla base criminalità, che è un suffissato nominale deaggettivale.

2.3.1.1.3. I suffissoidi -logia e -logo. Il sostantivo composto criminologia 'scienza che ha per oggetto lo studio dei crimini e dei criminali' e il sostantivo composto criminologo hanno come base il nome crimine, mentre il secondo elemento formativo è costituito dal suffissoide -logia in criminologia, rispettivamente dal suffissoide -logo in 
criminologo. I prefissoidi -logia e -logo si hanno ancora in mafiologia 'studio del fenomeno della mafia' e mafiologo 'studioso o esperto della mafia come fenomeno politico-sociale'. Alcuni di questi composti sono neologismi: criminologo, mafiologia, mafiologo, microcriminalità.

\subsubsection{I sostantivi composti ottenuti con due elementi colti}

\subsection{I suffissoidi-cida ${ }^{11}$ e-cidio}

Il suffissoide -cida 'chi uccide' forma i composti infanticida 'chi uccide un neonato', omicida ${ }^{12}$ 'chi ha ucciso una o più persone', patricida (o parricida) 'chi uccide il padre', uxoricida (o ussoricida) 'chi uccide la moglie'. Il suffissoide -cidio ha come significato 'uccisione' in infanticidio 'illecito penale di chi uccide un neonato', omicidio 'illecito penale di chi ha ucciso una o più persone', patricidio (o parricidio) 'illecito penale di chi uccide il padre', uxoricidio (o ussoricidio) 'omicidio aggravato in quanto commesso contro il coniuge'.

\subsubsection{I composti analitici. Unità lessicali sintagmatiche}

Sono composti formati dal nome e dall'aggettivo che determina il nome, da due nomi giustapposti o da due nomi uniti per mezzo di una preposizione.

\subsubsection{Il sostantivo accompagnato da un aggettivo}

I due elementi formativi lessicali sono posti accanto senza unirsi e fondersi insieme. Questi composti moderni sono molto numerosi: arresto domiciliare 'misura penale nell'obbligo di non abbandonare il proprio domicilio', assoluzione piena 'assoluzione per non aver commesso il fatto', legittima difesa 'uso della violenza fisica per difendersi o per difendere altri, consentito dalla legge', difesa eccessiva 'reato colposo di chi, per legittima difesa, reagisce in maniera eccessiva', giudice istruttore 'giudice sotto la cui direzione si svolge l'istruzione della causa', imputato recidivo 'chi dopo essere stato condannato per un reato ne commette un altro', ${ }^{13}$ istruzione sommaria 'istruzione dotata di formalità più semplici rispetto a quelle dell'istruzione formale'. ${ }^{14}$

11 Il suffissoide -cida forma anche composti appartenenti ad altri settori del linguaggio come insetticida, composto di insetto e -cida, che ha come significato 'sostanza impiegata per combattere gli insetti dannosi', o pesticida, che è un neologismo, formato sul modello inglese pesticide composto di pest 'pianta o animale dannoso' e -cide '-cida', che ha come significato 'prodotto chimico usato nella lotta contro gli organismi nocivi alle culture agricole'.

12 Il composto colto omicida si impiega anche come aggettivo: in arma omicida 'arma con cui si commette un omicidio'; in mano omicida 'mano che dà o ha dato la morte'.

13 L'unità lessicale imputato recidivo si usa anche come aggettivo sostantivato il recidivo; è un sostantivo formalmente identico all' aggettivo. Nel linguaggio giornalistico il sostantivo recidivo viene reso in croato con il nome 'ponavljač.

Cfr. altri esempi: carcerazione preventiva, circostanza aggravante, condanna condizionale, criminalità organizzata, custodia cautelare, delitto colposo, delitto doloso, detenzione domiciliare, diritto civile, diritto penale, diritto processuale, esecuzione sommaria, giurisprudenza cautelare, giusta causa, giustificato motivo, giustizia sommaria, illecito colposo, illecito penale, illiceità penale, inchiesta giudiziaria, indagine preliminare, interrogatorio sommario, istruzione preliminare, istruttoria preliminare, liberazione anticipata, liberazione condizionale, omicidio colposo, omicidio doloso, procedimento sommario, processo civile, processo penale, processo amministrativo, provvedimento cautelare, recidiva semplice, recidiva aggravata, recidiva 


\subsection{Il sostantivo accompagnato dal prefissato aggettivale}

Sono sostantivi composti formati dal nome e dall'aggettivo prefissato che sono posti accanto senza fondersi insieme: legge antimafia, commercio illecito, commissione antimafia, bene inalienabile, Direzione Distrettuale Antimafia, Direzione Nazionale Antimafia, diritto inalienabile, Giudice incompetente, mezzi illeciti, Procuratore Nazionale Antimafia.

\subsubsection{Due sostantivi giustapposti}

I due sostantivi sono accostati senza unirsi e fondersi insieme. Alcuni esempi: legge delega 'legge che il governo emana in base a delega del parlamento', legge ponte 'emessa in attesa di un'altra legge più organica e quindi migliore', legge quadro o legge cornice 'che contiene i principi fondamentali relativi all'ordinamento di una determinata materia giuridica', legge stralcio 'parte di una legge più generale non ancora approvata dal Parlamento'.

\subsubsection{Due sostantivi uniti per mezzo di una preposizione}

Questi composti moderni sono caratteristici del lessico giuridico: accesso sul luogo 'formalità processuale consistente in un sopralluogo al fine di reperire ogni possibile mezzo di prova', accuse senza fondamento 'accuse infondate', atto d'accusa 'quello che porta a conoscenza dell'imputato il reato a lui attribuito', stato d'accusa 'condizione di chi è imputato di reato', assoluzione per insufficienza di prove 'assoluzione piena', condanna senza appello 'condanna definitiva', confronto all'americana 'quello in cui il testimone deve riconoscere l'indiziato individuandolo tra altre persone di configurazione fisica simile', eccesso di difesa 'difesa eccessiva', negozio di accertamento 'negozio con il quale si accerta la situazione giuridica esistente tra le parti per eliminare ogni eventuale controversia', Registro degli indagati 'rubrica nella quale il pubblico ministero iscrive il nome delle persone sottoposte a indagine', tentativo di reato 'compimento di atti diretti a commettere un reato'. 15

\section{Gli aggettivi formati}

\subsection{I suffissati}

I suffissi aggiunti alle basi nominali, aggettivali e verbali formano aggettivi suffissati. Se teniamo conto della categoria di partenza del procedimento di suffissazione,

reiterata, sentenza assolutoria, sentenza parziale, sentenza definitiva, separazione consensuale, separazione giudiziale, soggiorno obbligato, tentato omiciio, tentata truffa, reato tentato.

Cfr. altri esempi: conflitto di competenza, conflitto di giurisdizione, contratto di lavoro, datore di lavoro, difensore di fiducia, difensore d'ufficio, diritto di sciopero, diritto alla libertà, diritto di seguito, diritto del lavoro, divieto di caccia, divieto di sosta, divieto di transito, divieto di soggiorno, Giudice per le Indagini Preliminari, Giudice dell'Udienza Preliminare, Giudici del Riesame, Tribunale del Riesame, Giudizio di delibazione, interrogatorio di garanzia, mandato o ordine di accompagnamento, mandato di arresto, mandato di cattura, obbligo di soggiorno, omissione di soccorso, ordine di cattura, posto di lavoro, prestatore di lavoro, processo di revisione, rapporto di lavoro, reato di adescamento, sentenza di assoluzione, sentenza di condanna, sentenza di conferma, sentenza di revisione, sequestro di persona, spaccio di stupefacenti, stato di necessità, violazione di corrispondenza. 
dei suffissi e della categoria di arrivo, possiamo parlare di due tipi di aggettivi suffissati riscontrati nel nostro corpus giuridico: suffissati aggettivali denominali e suffissati aggettivali deverbali.

\subsubsection{I suffissati aggettivali}

3.1.1.1. Gli aggettivi ottenuti con il suffisso-bile

Il suffisso -bile e le varianti morfonologiche -abile e -ibile formano numerosi aggettivi da basi verbali; i suffissati significano 'possibilità': abrogabile 'che si può abrogare', accertabile 'che si può accertare', annullabile 'che si può annullare', catturabile 'che può essere catturato', giudicabile 'che potrà $o$ dovrà essere oggetto di pronuncia giurisdizionale', indennizzabile 'che si può o si deve indennizzare', procedibile si dice di giudizio 'che può essere o può avere corso ulteriore', processabile 'che si può processare. ${ }^{16}$

I suffissati aggettivali deverbali abrogabile, accertabile, confrontabile, indennizzabile e procedibile sono neologismi.

\subsubsection{Gli aggettivi ottenuti con il suffisso -ale}

Il suffisso -ale e la variante morfonologica -uale formano i suffissati aggettivali da basi nominali: condominiale 'relativo a condominio', conflittuale 'di conflitto', 'caratterizzato da un conflitto', criminale 'che riguarda il crimine', delinquenziale 'da delinquente, della delinquenza', dibattimentale 'di/relativo a, dibattimento', giurisdizionale 'della, relativo alla, giurisdizione', giustiziale 'relativo alle norme, al potere e all'organizzazione della giustizia'. Dal nome pena si ha il suffissato penale, e dal suffissato aggettivale denominale penale si forma l'avverbio di modo o maniera in -mente: penalmente 'secondo le norme e i procedimenti del diritto penale'; dal nome procedimento si ha il suffissato procedimentale 'che riguarda un procedimento' e da processo si ha l'aggettivo suffissato processuale 'che concerne un processo' come in diritto processuale 'complesso degli atti legislativi che disciplinano il processo'.

I suffissati aggettivali denominali condominiale e conflittuale sono neologismi.

3.1.1.3. Gli aggettivi ottenuti con il suffisso-ivo

Il suffisso -ivo serve a formare aggettivi suffissati da basi verbali e anche da quelle nominali: abrogativo 'che abroga, che è atto ad abrogare', affidativo 'di affidamento, che concerne la consegna o la custodia in affidamento', assicurativo 'che concerne un' assicurazione', confutativo 'che vale a confutare', detentivo 'restrittivo della libertà personale', impugnativo 'che si riferisce all'impugnazione o che è diretto a impugnare', investigativo 'che tende o è atto a investigare', punitivo 'che tende o serve a punire'. L'aggettivo suggestivo (la cui base è costituita dal nome suggestione) 'che suggerisce la risposta' fa parte della terminologia giuridica: le domande suggestive sono proibite agli avvocati durante il processo.

Gli aggettivi suffissati affidativo e detentivo sono neologismi.

16 Cfr. altri suffissati aggettivali deverbali in -bile/-abile/-ibile: adescabile, affrancabile, alienabile, appellabile, assicurabile, confiscabile, confrontabile, confutabile, difendibile, imputabile, incolpabile, incriminabile, invalidabile, patteggiabile, punibile, querelabile, sequestrabile. 


\subsubsection{Gli aggettivi ottenuti con il suffisso -ario}

Il suffisso -ario forma aggettivi suffissati da basi nominali (e anche verbali): affidatario 'che ha in affidamento qualcuno o qualcosa', carcerario 'relativo alle carceri', indiziario 'che può valere come indizio' o 'che è fondato solo su indizi in mancanza di prove', indennitario 'che ha funzione di indennità o indennizzo'; dal nome penitenza si ha l'aggettivo penitenziario 'che concerne l'organizzazione delle istituzioni carcerarie ed è relativo all'espiazione di una pena detentiva'.

Gli aggettivi suffissati affidatario e indennitario sono neologismi.

3.1.1.5. Gli aggettivi ottenuti con il suffisso -orio

Il suffisso -orio forma gli aggettivi suffissati da basi verbali e da quelle nominali: abrogatorio 'che serve ad abrogare', accessorio si riferisce a 'bene connesso con un altro bene detto principale, di cui completa la funzione' come in diritto accessorio 'che è quello che viene trasmesso o acquisito insieme a un diritto principale'; l'aggettivo accusatorio definisce nel sistema processuale accusatorio un 'tipo di processo penale caratterizzato dalla parità tra accusa e difesa nell'individuazione e nella prova dei fatti rilevanti per il giudizio'; assolutorio ha come significato 'che assolve', confutatorio 'che ha lo scopo di confutare', inquisitorio 'di/da inquisizione': in un processo inquisitorio la ricerca dei fatti e delle prove è affidata a organi giudiziari, istruttorio 'che riguarda l'istruzione'. ${ }^{17}$

\subsubsection{Gli aggettivi ottenuti con il suffisso -istico}

La base dell' aggettivo suffissato civilistico 'concernente il diritto civile' è costituita dal sintagma diritto civile; dalla stessa base si forma l'avverbio di modo o maniera in -mente: civilmente 'secondo le norme del diritto civile; la base dell' aggettivo suffissato penalistico 'relativo al diritto penale', è costituita dal sintagma diritto penale; gli aggettivi in -istico civilistico e penalistico si connettono con i sostantivi in -ista: civilista e penalista. L'aggettivo colpevolistico ha come base l'aggettivo colpevole e ha come significato 'di colpevolismo o di colpevolista', l'aggettivo suffissato divorzistico ha come base il sostantivo divorzio e ha come significato 'del/relativo al divorzio', l'aggettivo giurisdizionalistico ha come base l'aggettivo in -ale, giurisdizionale e ha come significato 'relativo al o fondato sul giurisdizionalismo'. Gli agettivi in -istico: colpevolistico, divorzistico e giurisdizionalistico si connettono con i nomi in -ismo: colpevolismo, divorzismo, giurisdizionalismo e con i nomi in -ista: colpevolista, divorzista, giurisdizionalista.

Gli aggettivi suffissati civilistico, colpevolistico, divorzistico, giurisdizionalistico e penalistico sono neologismi.

\subsubsection{I suffissati aggettivali ottenuti con vari suffissi}

Il suffisso -oso forma aggettivi suffissati da basi nominali: dalla base colpa si ha colposo 'commesso per negligenza, imprudenza, imperizia, ma senza volontà di nuo-

17 L'aggettivo istruttorio s'impiega anche come sostantivo. L'aggettivo sostantivato di forma femminile, istruttoria, sottintende fase istruttoria. 
cere'; da crimine si ha criminoso 'che ha i caratteri di crimine'; da dolo si ha doloso 'commesso con dolo'; da mafia si ha mafioso 'tipico o caratteristico della mafia o che fa parte della mafia' ${ }^{18}$ Il suffisso -tore, proprio della suffissazione nominale, si trova anche in suffissati aggettivali deverbali: confiscatore 'che confisca', confutatore 'che confuta', punitore 'che punisce'. Il suffissato aggettivale denominale in -evole, colpevole, ha come base il nome colpa: 'che è in colpa', 'che ha commesso un reato'. Il suffisso aggettivale denominale -are si trova in cautelare che è un neologismo ed ha come significato 'che tende ad evitare un danno' e in domiciliare 'del domicilio'; il suffisso aggettivale denominale -esco si ha in avvocatesco 'da avvocato' con significato spregiativo; il suffisso -ile si ha in divorzile 'di, relativo a, divorzio'.

Nel corpus giuridico troviamo anche i participi presenti usati come aggettivi: sono aggettivi deverbali formalmente identici ai participi presenti: 1'aggettivo abberrante in reato abberrante 'commesso involontariamente o per errore durante il compimento di un altro reato', l'aggettivo accreditante 'che accredita e l'aggettivo scriminante ${ }^{19}$ 'che costituisce causa di giustificazione del reato' ${ }^{20}$

I participi presenti aggettivati abberrante, accreditante e scriminante sono neologismi.

\subsection{I prefissati}

I prefissi premessi a un nome, a un aggettivo o a un verbo formano i prefissati. Sono formati lessicali ottenuti con il procedimento di prefissazione senza mutamento di categoria.

\subsubsection{I prefissati aggettivali}

I prefissi premessi a un aggettivo formano gli aggettivi prefissati. Sono i prefissi anti-, co- e in-.

Il prefissato aggettivale antigiuridico è formato con il prefisso anti- che indica avversione, disposizione a contrastare; premesso all'aggettivo giuridico forma il prefissato aggettivale antigiuridico $o^{21}$ 'contrario a ciò che è riconosciuto o stabilito dal diritto'. Il prefisso -anti forma l'aggettivo invariabile antimafia 'finalizzato a combattere la mafia'. Il prefissato coimputato, aggettivo e nome, formato con il prefisso copremesso a imputato, indica unione, compagnia e ha come significato 'chi/che è imputato insieme ad altri'. Il prefisso in- e la variante morfonologica -il con valore negativo formano i prefissati aggettivali: illecito 22 'contrario alle norme giuridiche, morali e

18 Il suffisato aggettivale denominale mafioso si usa anche come sostantivo: il mafioso 'membro della mafia'.

Cfr. anche altri esempi: assicurante, accreditante, deliberante, delinquente, querelante.

20 Cfr. anche i participi passati usati come aggettivi; sono deverbali formalmente identici al participio passato: accertato, accusato, affrancato, arrestato, assicurato, carcerato, deliberato, detenuto, divorziato, giustiziato, imputato, incriminato, indagato, indiziato, tentato.

21 Dal prefissato aggettivale antigiuridico si forma l'avverbio di modo o maniera in -mente: antigiuridicamente.

Dal prefissato aggettivale illecito si forma l'avverbio di modo o maniera in -mente: illecitamente. 
religiose', inalienabile 'che non può essere trasferito ad altri', inamovibile 'che non può essere rimosso o trasferito arbitrariamente dall'ufficio o carica che riempie', incolpevole 'che è senza colpa', incompetente 'che non ha competenza'.

\subsection{I composti}

\subsubsection{I composti allogeni ottenuti con un elemento colto}

L'aggettivo composto criminogeno 'che genera o fornisce attività criminali' è costituito da due elementi formativi, dal nome crimine e dal suffissoide -geno che ha come significato 'che genera', 'che produce'.

\section{I verbi formati}

\subsection{I suffissati}

\subsubsection{I suffissati verbali}

I suffissati verbali si formano da basi nominali e da quelle aggettivali. Possiamo parlare di due tipi di aggettivi suffissati riscontrati nel nostro corpus giuridico: di suffissati verbali denominali e di suffissati verbali deaggettivali.

Da basi nominali si hanno i suffissati verbali denominali: assassinare 'uccidere un essere umano, specialmente per scopi criminali', catturare 'far prigioniero' da base viva cattura 'restrizione della libertà personale introduttiva della custodia cautelare', carcerare 'mettere in carcere', cautelare 'assicurare prendendo la dovuta cautela', controquerelare 23 'dare o sporgere controquerela contro qualcuno', decretare 'statuire con decreto', indagare 'sottoporre a indagine', indiziare 'indicare qualcuno come colpevole (o sospetto) in base a indizi', processare 'sottoporre a processo, specialmente penale', sequestrare 'disporre (o eseguire) un sequestro', 'porre sotto sequestro'. Da basi aggettivali si hanno i suffissati verbali deaggettivali; nella formazione di questi suffissati prevale il suffisso -izzare: colpevolizzare 'far sentire colpevole', criminalizzare 'considerare criminale ciò che giuridicamente non lo è', giurisdizionalizzare 'assoggettare a giurisdizione', invalidare 'rendere o dichiarare invalido' (un atto, un contratto), procedimentalizzare 'assoggettare a un atto procedimentale'. I suffissati verbali controquerelare, giurisdizionalizzare e procedimentalizzare sono neologismi.

\subsection{I prefissati}

\subsubsection{I prefissati verbali}

I prefissi $a$ - e $r$ - premessi a un verbo formano i verbi prefissati. Il prefisso $a$ - che indica avvicinamento, direzione, tendenza verso qualcosa forma il prefissato affidare 'dare in custodia', 'dare in affidamento'. Il prefisso ri- che esprime ritorno a una fase anteriore forma, insieme con il verbo assumere, il prefissato riassumere che in riassumere un lavoratore licenziato ha come significato 'accogliere come lavoratore qualcuno precedentemente già stato alle proprie dipendenze'.

23 Il suffissato verbale denominale controquerelare ha come base il prefissato controquerela. 


\subsection{I parasintetici}

Sono verbi derivati per parasintesi; più precisamente alla base si aggiungono simultaneamente il prefisso che precede la base e il suffisso che la segue.

La base del verbo accertare è costituita dall'aggettivo certo a cui si aggiungono simultaneamente due affissi: $a$ - che precede la base e -are che la segue; formano insieme il verbo parasintetico accertare che ha come significato 'riconoscere come certo'. Alla base del verbo transitivo affrancare, costituita dall'aggettivo franco, si aggiungono simultaneamente due affissi: $a$ - che precede la base e -are che la segue; il verbo parasintetico affrancare ha come significato 'rendere franco, libero un bene da pesi ed oneri che gravano su di esso'; il verbo parasintetico riflessivo affrancarsi ha come significato 'liberarsi da obbligazioni od oneri'. La base del verbo parasintetico imprigionare, costituita dal nome prigione, e due affissi aggiunti alla base: in-, che la precede e significa 'dentro', e -are che la segue, formano il verbo parasintetico imprigionare che ha come significato 'mettere o far mettere qualcuno in prigione'. La base del verbo parasintetico scarcerare è costituita dal nome carcere a cui si aggiungono simultaneamente due affissi: $s$-, che precede la base e indica separazione, allontanamento, e -are che segue la base; il verbo parasintetico scarcerare ha come significato 'liberare dal carcere'.

\subsection{I composti}

\subsubsection{I composti analitici. Unità lessicali sintagmatiche}

Sono composti moderni costituiti da un verbo e da un nome; il nome è di solito preceduto da una preposizione: costituirsi in giudizio 'nel processo civile, compiere le formalità richieste dalla legge per presentarsi ufficialmente alle altre parti e al giudice'; difendersi in giudizio 'sostenere le proprie pretese ribattendo le proprie accuse e/o imputazioni'; recedere da un contratto 'tirarsi indietro da un contratto'; soccombere in giudizio 'perdere una causa giudiziaria'; spacciare droga 'mettere in circolazione la droga in modo illecito'.

\section{Conclusione}

Il presente lavoro si propone di analizzare il linguaggio della giurisprudenza dal punto di vista della formazione delle parole nonché di mettere in rilievo i procedimenti formativi caratteristici del lessico della giurisprudenza. Il lessico giuridico, fissandosi da anni e per sua natura poco innovativo, si rigenera per vie interne mediante i meccanismi della suffissazione, della composizione e della prefissazione. La composizione delle parole è propria dei nomi; sono particolarmente numerosi i composti scientifici e i composti analitici, formati da un nome e da un aggettivo, da due nomi giustapposti o da due nomi uniti per mezzo di una preposizione. La suffissazione è propria dei nomi (in -tà, -mento, -tore, -ista, -zione, -ario, -ezza, -iere, -iero, -ura) e degli aggettivi (in -bile, -ale, -ivo, -ario, -orio, -istico, -oso, -evole). Nella suffissazione verbale prevalgono i suffissati verbali deaggettivali in -izzare. La prefissazione è caratteristica dei 
nomi (ottenuti con i prefissi contro-, in-, semi-, sopra-, super-) e degli aggettivi (ottenuti con i prefissi anti-, co-, in-). La formazione dei verbi riguarda i verbi parasintetici derivati per parasintesi e i composti analitici formati da un verbo e da un nome preceduto da una preposizione o senza preposizione.

Nel lessico della giurisprudenza convivono parole formate tradizionali e neologismi.

\section{Bibliografia}

Agostini, F., Simone, R., Vignuzzi, U. (a cura di) (1972), La grammatica. La lessicologia, Atti del I e II Convegno di Studi, SLI (Roma maggio 1967 e aprile 1968), Roma, Bulzoni.

ALINEI, M. (1962), Dizionario inverso italiano, The Hague.

ANć, V. (1998), Rječnik hrvatskoga jezika, Zagreb, Novi Liber.

BABIĆ, S. (1991), Tvorba riječi u hrvatskom književnom jeziku. Nacrt za gramatiku. HAZU, Zagreb, Globus.

BATTAGlia, S. (a cura di), (dal 1961 in poi), Grande dizionario della lingua italiana, Torino, UTET.

BeCCARIA, G. L. (1987), I linguaggi settoriali in Italia, Milano, Bompiani.

BeLVEDere, A., JoRI, M., LANTELlA, L. (1979), Definizioni giuridiche e ideologiche, Milano, Giuffrè Editore.

BERRUTO, G. (1995), Fondamenti di sociolinguistica, Roma-Bari, Laterza.

CAlleri, D., Marello, C. (a cura di) (1982), Linguistica contrastiva, Atti del XIII Congresso internazionale di Studi, SLI (Asti maggio 1979), Roma, Bulzoni.

Cortelazzo, M. (1994), Lingue speciali. La dimensione verticale. Studi linguistici applicati, Padova, Unipress.

Cortelazzo, M., Cardinale, U. (1988), Dizionario di parole nuove 1964-1987, Torino, Loescher.

DARDANO, M. (1978), La formazione delle parole nell'italiano d'oggi, Roma, Bulzoni.

DARDANO, M. (1986), Il linguaggio dei giomali italiani, Bari, Laterza.

Dardano, M., Glovanardi, C. (2001), Le strategie dell'italiano scritto, Bologna, Zanichelli.

De MaUro, T. (a cura di) (1994), Studi sul trattamento linguistico dell'informazione scientifica, Roma, Bulzoni.

DE MAURo, T. (1997), Guida all'uso delle parole, Roma, Editori Riuniti.

DE MAURO, T. (1998), Storia linguistica dell 'Italia unita, Bari, Laterza.

De MaUro, T. (1999), Grande Dizionario Italiano dell'Uso (Gradit), Torino, UTET, 6 volumi e CD-Rom.

DE MAURo, Lo CASCIO, V. (1997), Lessico e grammatica. Teorie linguistiche e applicazioni lessicografiche, Atti del convegno interannuale, SLI (Madrid febbraio 1995).

Dizionario Italiano Sabatini Coletti (1997), Edizione con CD-Rom, Firenze, Giunti Multimedia.

FABIAN, Zs., SALVI, G. (a cura di) (2001), Semantica e lessicologia storiche, Atti del XXXII Congresso internazionale di Studi, SLI (Budapest ottobre 1998).

Forcon, A. (1990), Dizionario delle nuove parole italiane, Milano, Sugarco.

Gambarara, D., RAMAT, P. (a cura di) (1977), Dieci anni di linguistica italiana (1965-1975), Roma, Bulzoni.

GARAVELli MONTARA, B. (2001), Le parole e la giustizia. Divagazioni grammaticali e retoriche su testi giuridici italiani, Torino, Einaudi.

GARZANTI ED. (1998), Il Grande Dizionario Garzanti della lingua italiana, Milano.

GotrI, M. (1991), I linguaggi specialistici, Firenze, La Nuova Italia.

Gremas, A. J. (2000), Semantica strutturale, Roma, Meltemi.

LEON, F.A., De BIASI, N. (a cura di) (1979), Lessico e Semantica, Atti del XII Congresso internazionale di Studi, SLI (Sorrento maggio 1978), Roma, Bulzoni.

Lo DuCA, M. G. (1990), Creatività e regole. Studio sull'acquisizione della morfologia derivativa dell'italiano, Bologna, Il Mulino.

LuzzaTI, C. (1990), La vaghezza delle norme. Un'analisi del linguaggio Giuridico, Milano, Giuffrè Editore.

MARCHAND, H. (1969), The Categorie and Types of Present-Day English Word-Formation, München.

MARELLO, C. (1996), Le parole dell' italiano. Lessico e dizionari, Bologna, Zanichelli.

MARTINET, A. (1967), Eléments de linguistique générale, Paris, Armand Colin.

MedicI, M. (1965), La lingua delle pagine gialle, Torino.

MIGLIORNI, B. (1965), Vocabolario della lingua italiana (Edizione rinnovata del Vocabolario della lingua italiana di G. Cappuccini e B. Migliorini), Torino. 
Migliorni, B. (1988), Storia della lingua italiana. Introduzione di G. Ghinassi, vol. I-II, Firenze, Sansoni. Migliorini, B., BALDELli, I. (1981), Breve storia della lingua italiana, Firenze, Sansoni.

Mroni, A. M., Cortelazzo, M. A. (a cura di) (1992), La linguistica italiana degli anni 1976-1986, Roma, Bulzoni.

Mucciante, L., Telmon, T. (a cura di) (1997), Lessicologia e lessicografia. Atti del Convegno della Società Italiana di Dialettologia, Roma.

Petralli, A. (1996), Neologismi e nuovi media. Verso la "globalizzazione multimedia" della comunicazione? Bologna, Clueb, Collana Alma materiali.

Potrier, B. (1992), Sémantique générale, Paris, PUF.

PRATI, A. (1958), Nomi composti con verbi, in "Revue de Linguistique Romane" XXII, Paris, pp. 98-119.

RENZI, L. (a cura di) (1988), Grande grammatica italiana di consultazione, vol. I, La frase. I sintagmi nominale e preposizionale, Bologna, II Mulino.

RENZI, L., SALVI, G. (a cura di) (1991), Grande grammatica italiana di consultazione, vol. II, La frase. I sintagmi verbale, aggettivale, avverbiale, Bologna, Il Mulino.

Renzi, L., SAlvi, G., CARDINALETTI, A. (a cura di) (1995), Grande grammatica italiana di consultazione, vol. III, Tipi di frasi, deissi, formazione delle parole, Bologna, Il Mulino.

RoHLFs, G. (1969), Grammatica storica della lingua italiana e dei suoi dialetti. III, Sintassi e formazione delle parole (trad. it.), Torino, Einaudi.

Quarantorto, C. (1987), Dizionario del nuovo italiano, Roma, Newton Compton.

SCHIAFFINI, A. (1963-64), La formazione del lessico italiano, dispense universitarie, Roma.

SCHWARZE, C. (2001), Introduction à la sémantique lexicale, Tübingen, Narr.

SERIANNI, L. (1988), Grammatica italiana. Italiano comune e lingua letteraria, Torino, UTET.

SERIANNI, L., TRIfoNe, P. (1994), Storia della lingua italiana, vol. II, Scritto e parlato, Torino, Einaudi.

SOBRERo, A. (a cura di) (1993), Introduzione all 'italiano contemporaneo. Le strutture, Roma-Bari, Laterza.

TEKAVČIĆ, P. (1980), Grammatica storica dell'italiano, Vol. III: Lessico, Bologna, Il Mulino.

TOllemache, F. (1978), id. Deverbali, in Enciclopedia Dantesca, vol. VI, Roma, Istituto della Enciclopedia Italiana.

VuČETIĆ, Z. (1998), Formazioni scientifiche. Primi materiali, in "Linguistica" XXXVIII, 2, Ljubljana, pp. 167-182.

VUČETIĆ, Z. (1999), Contributo allo studio della composizione delle parole. Raffronto contrastivo italiano-croato, croato-italiano. Primi risultati, in "Linguistica" XXXIX, Ljubljana, pp. 83-98.

VuČETIĆ, Z. (2001), La terminologia marinaresca studiata dal punto di vista della formazione delle parole, in "Linguistica" XLI, Ljubljana, pp. 111-128.

ZINGARELli, N. (2002), Vocabolario della lingua italiana, Bologna, Zanichelli.

\section{Povzetek}

\section{PRAVNIŠKI JEZIK V LUČI BESEDNE TVORBE}

Prispevek obravnava in razčlenjuje italijanski pravniški jezik glede na besedno tvorbo. Predstavlja postopke, ki jih je mogoče ugotoviti v pravniškem jeziku; ta se bogati s sestavljanjem besed, a tudi s tvorbo, bodisi s pomočjo pripon ali predpon.

Sestavljanje besed je značilno za samostalnike; gre za učene tvorbe s prvinami grškega in latinskega izvora, a tudi za moderne analitične sestavljenke samostalnika in pridevnika ali pa dveh samostalnikov. Tvorba s pripono je tipična za samostalnike (pripone so -ta, -mento, -tore, -ista, -zione, -ario, -ezza, -iere, -ueri, -ura) in za pridevnike (pripone so -bile, -ale, -ivo, -ario, -orio, -istico, -oso, -evole). $\mathrm{V}$ tvorbi glagola s pomočjo pripone prevladujejo glagoli iz pridevniških osnov s pripono -izzare. Predponska tvorba je značilna za samostalnike (predpone so contro-, in-, semi-, sopra-, super-) in za pridevnike (tvorjene s predponami anti-, co-, in-). Za glagol pa se posebej ugotavljajo parasintetične zloženke, tvorjene iz glagola in samostalnika s predlogom ali brez njega. 